\title{
Cultural Differences in Belief Bias Associated with Deductive Reasoning?
}

\author{
Sara J. Unsworth, Douglas L. Medin \\ Department of Psychology, Northwestern University
}

Received 28 January 2004; received in revised form 17 August 2004; accepted 8 September 2004

\begin{abstract}
Norenzayan, Smith, Jun Kim, and Nisbett (2002) investigated cultural differences in the use of intuitive versus formal reasoning in 4 experiments. Our comment concerns the 4th experiment where Norenzayan et al. reported that, although there were no cultural differences in accuracy on abstract logical arguments, Koreans made more errors than U.S. undergraduates in judging the logical validity of concrete arguments. Norenzayan et al. concluded that Koreans are less likely than European Americans to decontextualize an argument's content from its logical structure, as Koreans were more likely to consider the believability of the conclusion when assessing an argument's validity (a belief bias). Notably, Korean participants were more conservative (less likely to say an argument is valid) than European American participants when assessing arguments. An analysis of the average of the hit and correct rejection rates in each of the conditions (abstract, concrete-believable, concrete-nonbelievable) revealed that, contrary to conclusions of Norenzayan et al., European Americans were no better than Koreans at determining the validity of concrete deductive arguments with conclusions varying in believability.
\end{abstract}

Norenzayan, Smith, Jun Kim, and Nisbett (2002) investigated whether there are cultural differences between East Asians, Asian Americans, and European Americans in their preferences for formal versus intuitive reasoning. Based on results from four experiments, the authors concluded that East Asians were less likely than European Americans to abandon intuitive reasoning (i.e., reasoning based on experience and information from the senses) in favor of formal reasoning (i.e., reasoning based on rules and formal logic).

Norenzayan et al.'s (2002) Experiment 4 provides the most direct test of their claim that cultural differences are observed in deductive reasoning. In Experiments 1 and 2 Norenzayan et al. examined categorization and conceptual structure, rather than deductive reasoning, and although Experiment 3 involved an evaluation of syllogisms, all of the syllogisms were valid, and participants were asked simply to rate the convincingness of the arguments rather than to assess the arguments' validity. In Experiment 4, participants were asked to make judgments

Requests for reprints should be sent to Sara Unsworth, Department of Psychology, Northwestern University, 2029 Sheridan Road, Evanston, IL 60208. E-mail: s-unsworth@ northwestern.edu 
about the validity of syllogisms, and the question of interest was whether Korean participants would be more influenced than European American participants by the believability of the conclusion of an argument when making such judgments. In general, Norenzayan et al. predicted that both cultural groups would be more likely to say that a valid argument is invalid if the conclusion is not believable (relative to when the conclusion is believable), and more likely to say that an invalid argument is valid if the conclusion is believable (relative to when the conclusion is not believable). Such differences are known as the belief bias effect. However, they also predicted that Korean participants would exhibit the belief bias effect to a greater extent than European Americans, because Koreans would be less likely to decontextualize the logic of an argument from the believability of the conclusion.

There were three conditions in this experiment: a condition in which arguments were abstract and lacking content (involving letters and foreign words rather than familiar words), a condition in which arguments were concrete (using familiar words) with believable conclusions, and a condition in which arguments were concrete with nonbelievable conclusions. In each condition, arguments were either valid or invalid.

The authors reported that in the abstract condition, Koreans were generally less likely than European Americans to judge an argument as valid, but when false alarms (percentage of "yes" responses for invalid arguments) were subtracted from hits (percentage of "yes" response for valid arguments), Korean and European American participants did not differ in accuracy. According to the authors, these results indicated that these groups do not differ in their logical reasoning ability independent of content. In addition, the authors reported that overall, both Korean and European American participants exhibited the belief bias effect. However, for valid arguments (but not for invalid arguments), the difference in "yes" responses between arguments with believable and nonbelievable conclusions was reliably greater for Koreans than for European Americans (see Table 1). They attributed this latter finding to a cultural difference in belief bias.

A closer examination of the data, however, reveals that the findings reported for Experiment 4 (Norenzayan et al., 2002) may be misleading because response bias needs to be addressed. Indeed, the authors noted that there was a significant response bias in all of the conditions in this experiment, such that Korean participants were significantly less likely than European American participants to indicate that an argument was valid (i.e., significantly less likely to respond "yes"). Although this result may be an interesting cultural difference on its own, the

Table 1

Percentage of "valid" responses for valid and invalid arguments for each condition as a function of culture

\begin{tabular}{llll}
\hline & Abstract & $\begin{array}{l}\text { Concrete } \\
\text { Nonbelievable }\end{array}$ & $\begin{array}{l}\text { Concrete } \\
\text { Believable }\end{array}$ \\
\hline Valid arguments & & & \\
$\quad$ Korean & 77.78 & 62.88 & 86.87 \\
$\quad$ European American & 85.06 & 76.72 & 91.95 \\
Invalid arguments & & & 17.85 \\
$\quad$ Korean & 29.29 & 9.50 & 27.01 \\
$\quad$ European American & 39.94 & 16.09 & \\
\hline
\end{tabular}


reason for the difference in response bias is unclear, and such a difference casts doubt on Norenzayan et al.'s conclusions for this experiment.

If Korean participants have a greater belief bias, then they should be more likely to ignore the logic of the arguments and make validity judgments on the basis of content (i.e., the believability of the conclusion). If this is the case, then the overall percentage of correct scores should be lower for Korean participants than for European American participants in the concrete nonbelievable and concrete believable conditions.

To test this prediction, we averaged the hit and correct rejection rates in each of the conditions (abstract, concrete believable, and concrete nonbelievable) for the two cultural groups in Experiment 4 of Norenzayan et al. (2002) and conducted a 2 (culture: Korean, European American) $\times 3$ (condition: abstract, concrete believable, concrete nonbelievable) analysis of variance. The hit and correct rejection rates, along with the averages of these rates in each condition for each cultural group, can be seen in Table 2. Results showed that the interaction between culture and condition approached significance, $F(2,368)=3.02$, mean standard error $[S E]=154.15, p=.05$. However, $t$ tests revealed that cultural differences were not significant in the abstract condition, $t<1$, in the concrete believable condition, $t<1$, or in the concrete nonbelievable condition, $t(184)=1.55, S E=2.35, p=.12$. Results also showed that there was a main effect of condition, $F(2,368)=30.59$, mean $S E=154.15, p<.001$, such that overall, values were highest in the concrete believable condition (83.56) and lowest in the abstract condition (73.45), with values in the concrete unbelievable condition falling in the middle (78.39). There was no main effect of culture, $F<1$.

These results suggest that when judging the validity of an argument, European Americans' accuracy is not significantly different from Koreans' accuracy, regardless of the believability of the conclusion. In addition, there appears to be a main effect of condition, such that participants were better able to discriminate between valid and invalid arguments when the arguments were concrete relative to when they were abstract, and when the conclusions were believable relative to when they were nonbelievable. Thus, it appears that the cultures do not differ in the extent to which they are influenced by the believability of the conclusion when assessing an argument's validity.

Table 2

Percentage of hits for valid arguments, percentage of correct rejections for invalid arguments, and average of hits and correct rejections for each condition as a function of culture

\begin{tabular}{llll}
\hline & Abstract & $\begin{array}{l}\text { Concrete } \\
\text { Nonbelievable }\end{array}$ & $\begin{array}{l}\text { Concrete } \\
\text { Believable }\end{array}$ \\
\hline Hits for valid arguments & & & \\
$\quad$ Korean & 77.78 & 62.88 & 86.87 \\
$\quad$ European American & 85.06 & 76.72 & 91.95 \\
Correct rejections for invalid arguments & & & 82.16 \\
$\quad$ Korean & 70.71 & 90.51 & 72.99 \\
$\quad$ European American & 60.06 & 83.91 & 84.51 \\
Average of hits and correct rejections & & & 82.47 \\
$\quad$ Korean & 74.24 & 76.69 & \\
$\quad$ European American & 72.56 & 80.32 & \\
\hline
\end{tabular}


When there are potential differences in response bias, it often is useful to employ signal detectability theory (SDT). Although SDT has not typically been used in the study of deductive reasoning, Klauer, Musch, and Naumer (2000) claimed that theories of belief bias, such as the selective scrutiny model (e.g., Evans, 1989) and the mental models account (e.g., Newstead, Pollard, Evans, \& Allen, 1992) imply that belief bias may be a result of response bias and that such response bias is analogous, if not equivalent, to the response bias defined in Green and Swets' (1974) signal detection model. A complication, however, is that there are a number of distinct ways in which response bias might operate (Klauer et al., 2000), and we do not have independent evidence favoring one form over others. ${ }^{1}$

Our analysis of the hit and correct rejection rates points to a different conclusion than that given by Norenzayan et al. (2002), at least with respect to their Experiment 4 . Norenzayan et al. concluded that Koreans are more likely to be influenced by the believability of conclusions when evaluating the validity of arguments and that this cultural difference was due to "differing tendencies to decontextualize the content of an argument from its logical structure" (p. 678). However, when overall accuracy is examined in each of the three conditions, the differences disappear. In short, although it may be possible that East Asians and Europeans use different cognitive strategies in other cognitive tasks, there is no evidence that Koreans show more belief bias than European Americans in deductive reasoning.

\section{Notes}

1. Nonetheless, for completeness we also employed a standard form of SDT to separate response-bias effects from discriminability, in this case between valid and invalid logical arguments (see Green \& Swets, 1974). Analyses using the group means reported in Norenzayan et al.'s (2002) Experiment 4 compared the hits and false alarms in each of the three conditions. (Note: Higher d' values indicate greater discriminability between hits and false alarms.) The results revealed no cultural differences in discriminability for either abstract or concrete arguments. European Americans scored only slightly higher than Koreans on concrete arguments with nonbelievable conclusions (1.71 vs. 1.67 , respectively), but this trend was reversed for concrete arguments with believable conclusions (2.02 and 2.08, respectively) and for abstract arguments (1.29 and 1.32, respectively). We also analyzed individual data using $\mathrm{A}^{\prime}$ as a measure of discrimination and found essentially the same results. Overall, these results provide converging support to the claim that the cultures do not differ in the extent to which they are influenced by the believability of the conclusion.

\section{Acknowledgments}

The authors thank Ara Norenzayan for graciously sending us the data from Experiment 4 in Norenzayan et al. (2002). We also would like to thank Peter Vishton and Satoru Suzuki for their advice. These efforts were supported by NSF Grant 9983620 to Medin. 


\section{References}

Evans, J. St. B. T. (1989). Bias in human reasoning: Causes and consequences. Hillsdale, NJ: Lawrence Erlbaum Associates, Inc.

Green, D. M., \& Swets, J. A. (1974). Signal detection theory and psychophysics. Huntington, NY: Krieger.

Klauer, K., Musch, J., \& Naumer, B. (2000). On belief bias in syllogistic reasoning. Psychological Review, 107, 852-884.

Newstead, S. E., Pollard, P., Evans, J. St. B. T., \& Allen, J. L. (1992). The source of belief bias effects in syllogistic reasoning. Cognition, 45, 257-284.

Norenzayan, A., Smith, E. E., Jun Kim, B., \& Nisbett, R. E. (2002). Cultural preferences for formal versus intuitive reasoning. Cognitive Science, 26, 653-684. 